\title{
Complementary split-ring resonator-coupled traveling wave accelerating structure
}

\author{
I. McGregor and K. M. Hock \\ Department of Physics, University of Liverpool, Liverpool L69 7ZE, United Kingdom \\ and Cockcroft Institute, Daresbury Laboratory, Warrington WA4 4AD, United Kingdom
}

(Received 14 March 2013; published 19 August 2013)

\begin{abstract}
In this paper, we present theoretical and simulation-based analyses of a novel, normal-conducting, multiple-cell, traveling wave accelerating structure. Instead of the conventional circular apertures, we utilize asymmetric complementary split-ring resonators to couple pillbox cavities and bring the phase velocity below that of the speed of light in vacuo. We show that this architecture exhibits a low, negative, group velocity and that the 0 through $\pi$ modes decrease in order of frequency-in contrast to conventional electrically coupled structures in which the 0 mode has the lowest frequency and the $\pi$ mode the highest. We illustrate the efficacy of the proposed design via electromagnetic and particle simulation results for a four-cell structure operating around $1.9 \mathrm{GHz}$. Results are given for operation in the $\pi, 2 \pi / 3$, and $\pi / 3$ modes. Our design achieves accelerating gradients of around $3.3 \mathrm{MV} / \mathrm{m}$ and a cavity voltage of $0.594 \mathrm{MV}$ for an applied rf power of $82 \mathrm{~kW}$ ( $\pi$ mode). The accelerating gradients achieved are up to 3.3 times that of a conventional circular aperture-coupled design with the same phase velocity, rf excitation power, operating frequency, mode type, and number of cells.
\end{abstract}

DOI: 10.1103/PhysRevSTAB.16.080102

PACS numbers: 29.20.- c, 81.05.Xj

\section{INTRODUCTION}

Circular waveguides propagating TM modes cannot be used to accelerate charged particles as they travel more slowly than wave fronts in the accelerating field, i.e., the phase velocity is greater than the speed of light in a vacuum. This means that a particle bunch slips behind the wave fronts and eventually experiences decelerating fields. However, as is well known, obstructions, in the form of circular irises, periodically placed in the waveguide, can lower the phase velocity to below that of the speed of light in vacuo [1] and, under such circumstances, the particles can be synchronized to the accelerating field and no longer slip behind the wave fronts. This paper presents a novel twist on this old idea, namely, the traditional circular irises are replaced with metamaterial unit elements. In particular, we use asymmetric complementary split-ring resonators (CSRRs) with a circular hole in the central metallization section to allow particle bunches to pass through. In contrast to conventional traveling wave structures utilizing electrical coupling between cells, when resonant coupling via CSRR metamaterial elements are used, the 0 to $\pi$ operating modes occur in order of decreasing frequency and the structure exhibits a negative group velocity. It will be shown that, for a given applied rf power, comparatively high accelerating gradients can be achieved.

The split-ring resonator (SRR), first described by Pendry [2], was suggested as a means of obtaining an effective

Published by the American Physical Society under the terms of the Creative Commons Attribution 3.0 License. Further distribution of this work must maintain attribution to the author(s) and the published article's title, journal citation, and DOI. macroscopic negative permeability from unit elements composed of these resonators. Arrays of SRRs may be combined with arrays of metallic wires or complementary split-ring resonators to form bulk metamaterials exhibiting simultaneous, effective, negative electrical permittivity and magnetic permeability. Such metamaterials exhibit unusual electromagnetic characteristics which are not found in naturally occurring electromagnetic media, including reverse-orientated Cerenkov radiation, inverse Doppler shift and negative refraction [3]. Owing to these interesting properties, Metamaterials have received much attention in fields as diverse as electromagnetic cloaking [4], traveling wave amplification at microwave and mm-wave frequencies [5,6], and particle accelerator technology [7].

CSRRs are normally used as metamaterial elements in order to provide an effective negative permittivity [8]. They are "complementary" to the better known SRRs in the sense that, where in the SRR there is metallization, there exists, in the CSRR, an absence of metallization and vice versa. They are the circuit dual of SRRs in the sense that SRRs have a predominantly magnetic response and yield negative effective permeability and CSRRs have a predominantly electric response and yield negative effective permittivity.

Some work has been done by other researchers to apply metamaterial concepts to particle accelerator technology. For example, the authors of [9] describes the indirect measurement of reverse directed wakefields created by an electron beam traversing a metamaterial-loaded waveguide. Other work on applying metamaterial concepts to particle accelerator technology has included a theoretical structure consisting of a "sandwich" of sheets of metal 


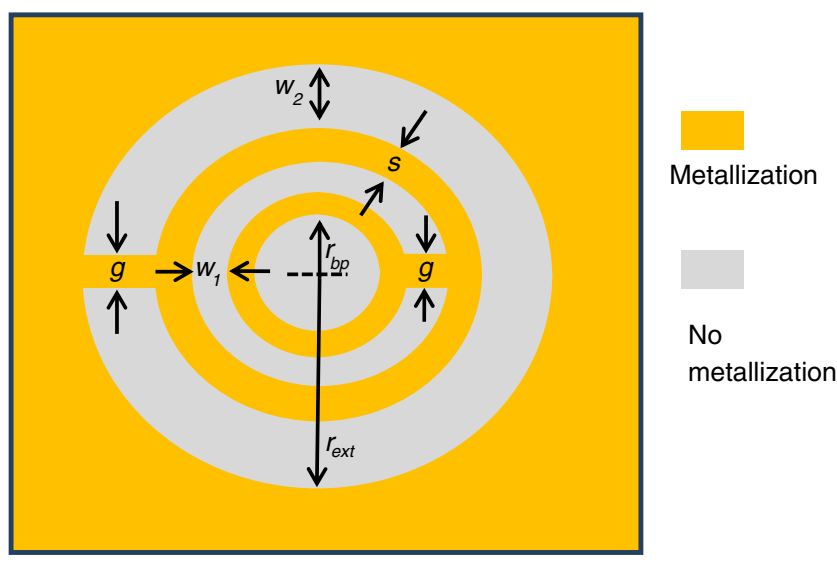

FIG. 1. Top view of the CSRR.

into which CSRRs are etched [5,10]. However, there is no indication that this structure is practical as no details of the feeding mechanism are given, magnetic walls are required as terminations to make the desired accelerating mode propagate, and simulations reveal that the peak surface electric fields are so high that only very modest powers can be applied before the onset of electron field emission. Some simulation-based studies have also been done on combining photonic crystals with metamaterials for $\mathrm{THz}$ sheet beam devices [11] without, however, demonstrating that particles can be accelerated using such methods. The work detailed in this paper, therefore, represents the first practical application of metamaterial concepts to particle accelerating structures. However, despite the negative group velocity, the electromagnetic environment inside our structure does not exhibit "left-handed" electromagnetic (EM) propagation. It does exhibit negative effective permittivity but, due to the absence of magnetic resonant elements, does not constitute a left-handed medium.

The paper is organized as follows. First, we briefly describe the properties of CSRRs. We then outline the geometry and operation of the accelerating structure. This is followed by a simple and novel analysis of the asymmetric CSRR's circuit dual-the asymmetric SRR.
A prior use of asymmetric CSRRs, or indeed, SRRs in the literature is unknown to the authors. They are used here because the coupling between cells and strength of the on-axis $E$ field can be modified by making the CSRRs asymmetric. The results of the CSRR analysis are then built upon and eventually used to compute the mode frequencies of the circular waveguide-coupled cells. In this latter analysis we use Slater's perturbation theorem to analyze the mode frequency shift which occurs when the metamaterial irises are introduced to the circular waveguide and to obtain the dispersion curve for an infinite chain of the unit cells. We compare this result and the group and phase velocities with those obtained from an eigenmode analysis using CST microwave studio. This is followed by detailed EM analyses and particle simulations (both obtained from CST) for a four-cell structure operating around $1.9 \mathrm{GHz}$. Finally, we compare the performance of our design with that of an equivalent conventional circular aperture-coupled traveling wave structure.

\section{DESCRIPTION OF THE TRAVELING WAVE STRUCTURE}

We begin by describing the geometry of the CSRR. Figure 1 shows a top view. The metal has a thickness $t$ into the page. It can be seen that the uniform portions of the structure resemble an asymmetric coplanar waveguide transmission line with ground-to-ground spacing $w_{1}+s+$ $w_{2}$ and a signal trace of width $s$. There are also two shunt inductive connections to the effective ground planes on the west and east side of the structure. When excited by an $E$ field pointing into the page, at a particular frequency dependent on the geometry of the CSRR, a resonant electric monopole is created with magnified field dependent on the structure's quality factor-also pointing into the plane of the structure but with opposite polarity to the excitation field.

Figure 2 shows a perspective and cut-plane view of the proposed accelerating structure. It can be seen to consist of a circular waveguide of radius $r_{c w}$ divided into, in this particular case, four cells of length $l$ coupled together by

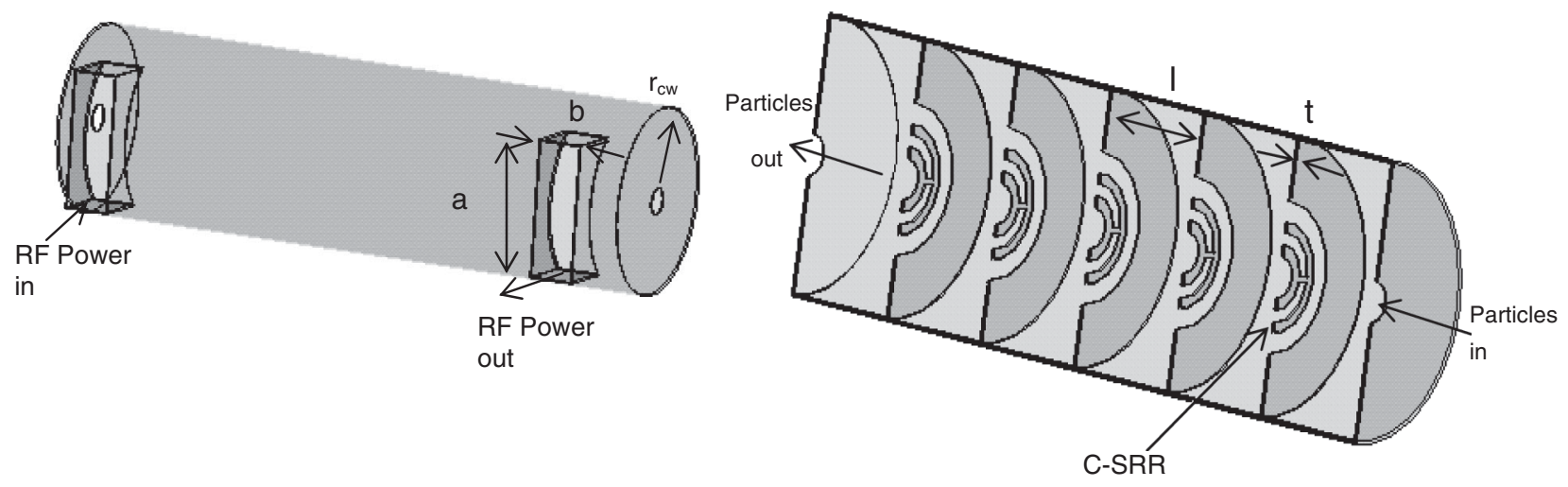

FIG. 2. Perspective and cut-plane view of the traveling wave structure. 

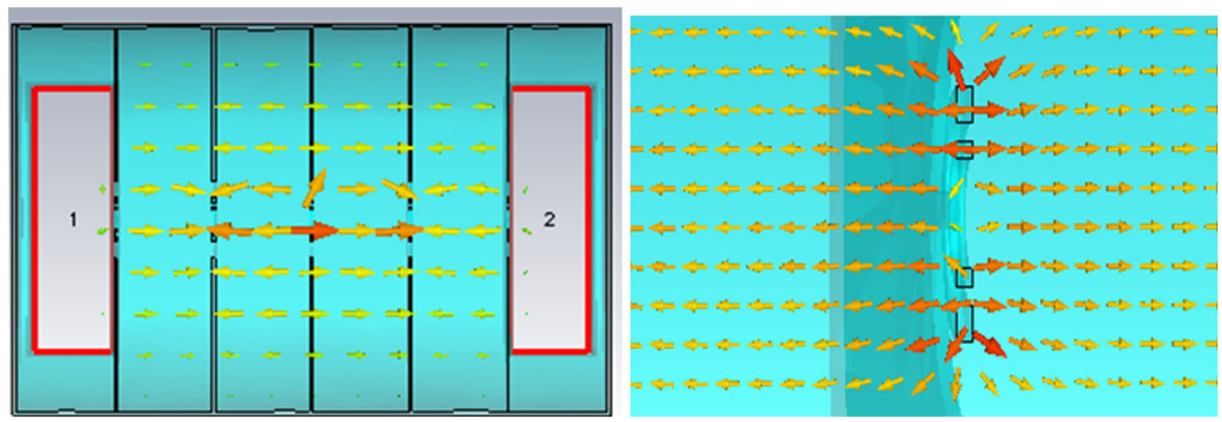

FIG. 3. $\quad E$-field distributions in the complete structure (left) and near one of the CSRR irises (right) for the $\pi$ mode.

the asymmetric CSRRs. Two cells at either extremity of the structure, also of length $l$, allow power to be injected and removed from the structure via rectangular waveguides of dimensions $a \times b$. These waveguides propagate the dominant $\mathrm{TE}_{10}$ mode. The input microwave power may be coupled to these rectangular waveguides through any standard high power mode launcher. The particle bunch is injected into the middle of the circular wall furthest from the source of rf power, traverses the structure via the holes in the CSRR inner "ground plane," and exits the structure through the middle of the outer circular wall furthest from the rectangular waveguide that removes the rf power.

Figure 3 shows 2D cuts of the electric field distributions in the complete structure and near one of the CSRR irises for the $\pi$ mode.

\section{ANALYSIS OF THE MODE FREQUENCIES}

We now analyze the mode frequencies of the structure. First, it is necessary to analyze the properties of the CSRRs themselves. An analysis of the structure can most easily be accomplished by consideration of the dual structure- the asymmetric SRR. This is possible due to the duality between the CSRR and the SRR [3]. This geometry is shown in Fig. 4. Strictly speaking, the duality is only valid

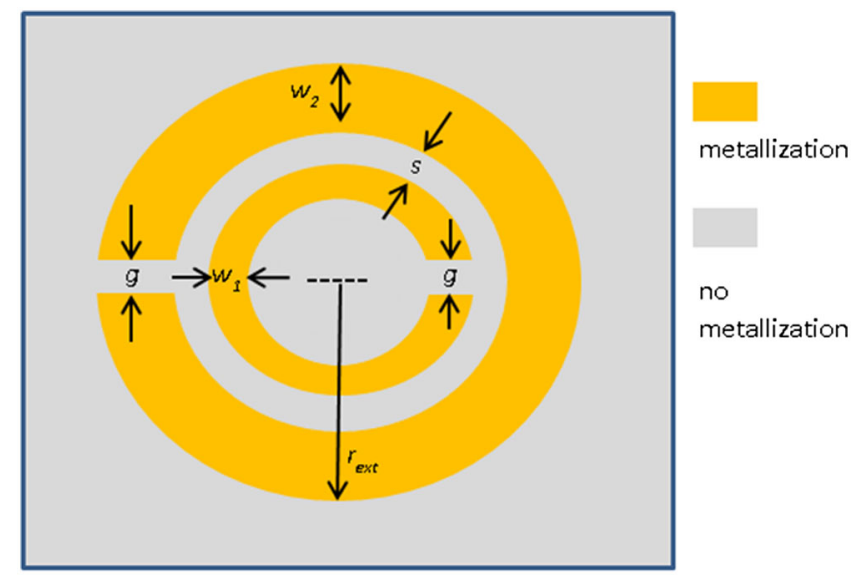

FIG. 4. Top view of the structure complementary to the CSRR: the SRR. for infinitesimally thin metallizations but, as will become apparent, this approximation is accurate enough for our purposes.

Figure 5 shows 2D cuts of typical $E$ and $H$ field distributions for the SRR and CSRR. The horizontal direction of the plots is into the page with reference to Figs. 1 and 4. The vertical direction of the plots is the same as in Figs. 1 and 4.

The upper and lower half of the SRR can be thought of as two separate coplanar strip (CPS) transmission lines connected in series. Thus, the effective capacitance of the structure is one quarter of the total distributed capacitance and the effective inductance is that of the entire structure.

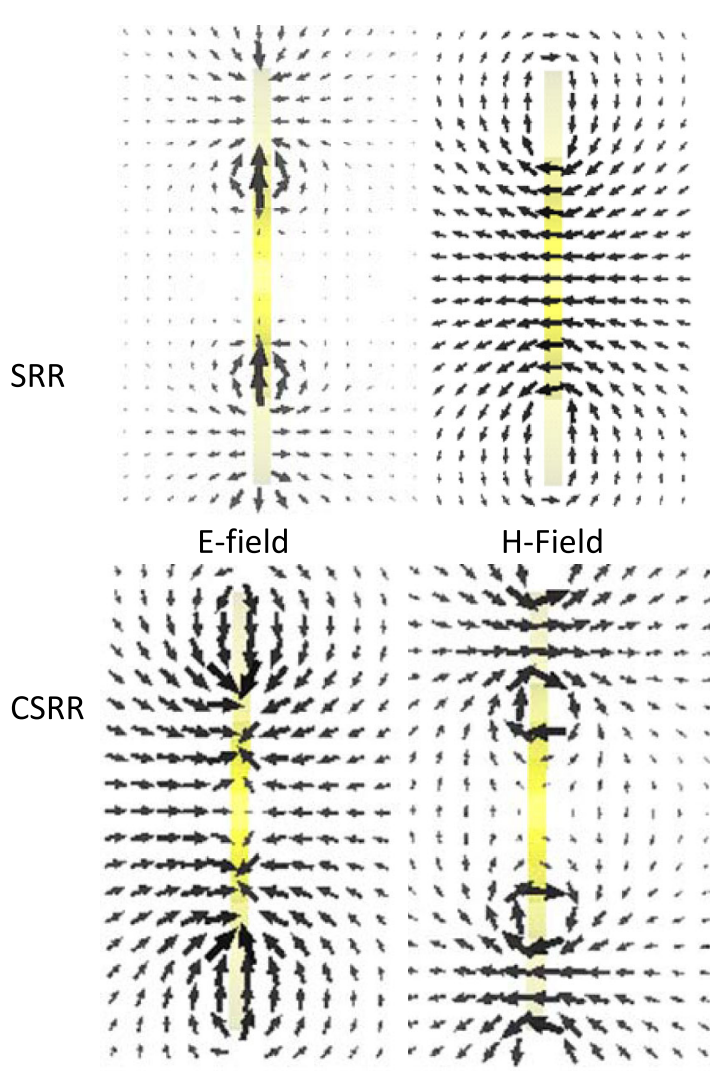

FIG. 5. Sketches of the magnetic (left) and electric (right) fields of the SRR (top) and CSRR (bottom) at resonance. 
Such an analysis is termed "quasistatic" as the frequency dependence is effectively ignored and the field distributions of the CPS-like sections are taken to be the same as if $\mathrm{dc}$ voltages and currents were present.

The per-unit-length capacitance of an asymmetric coplanar stripline can be calculated from [12]

$$
C_{\mathrm{pul}}=4 \varepsilon_{0}\left(\frac{K\left(k^{\prime}\right)}{K(k)}+\frac{1}{4} \frac{t}{s}\right) .
$$

$K$ (modulus) is a complete elliptical integral of the first kind. Here we have added an additional term-the second in the brackets - to account for the increased capacitance due to the finite metal thickness, $t$, of the effective transmission line. Of course, this is only an approximation as, in reality, the electric fields of the effective parallel plate capacitor formed by the metal thickness and the fields due to the top and bottom faces of the effective transmission line are not independent. However, an exact analysis would require numerical solutions to the conformal map of the structure. It will be shown, however, that this approximation is accurate enough for our present purposes. The complementary elliptic modulus is given by

$$
k^{\prime}=\sqrt{\frac{w_{1}}{w_{1}+s} \frac{w_{2}}{w_{2}+s}},
$$

and the normal modulus by

$$
k=\sqrt{1-k^{\prime 2}} .
$$

From basic transmission line theory, the per-unit-length inductance is given by

$$
L_{\mathrm{pul}}=\frac{1}{c^{2} C_{\mathrm{pul}}},
$$

where $c$ is the speed of light in a vacuum. The effective radius of the resonator is given by (see Fig. 3)

$$
r=r_{\mathrm{ext}}-w_{2}-s / 2 .
$$

We can also define an average radius as

$$
r_{\mathrm{av}}=\frac{2 r_{\mathrm{ext}}-w_{2}-s-w_{1}}{2} .
$$

These are the radii we must use to calculate the circumferences that multiply the per-unit-length parameters to give the lumped values. The lumped values are given by the following two equations:

$$
\begin{gathered}
C=\frac{\theta r C_{\text {pul }}}{4}+C_{\text {gap } 1}+C_{\text {gap } 2}, \\
L=\theta r_{\text {av }} L_{\text {pul }}+L_{\text {gap } 1}+L_{\text {gap } 2} .
\end{gathered}
$$

Here, $\theta$ is the effective angle covered by the uniform transmission line section. It differs from $2 \pi$ due to the presence of the "gaps" and is given, approximately, by

$$
\theta \approx 2 \pi-\frac{g}{r_{\mathrm{ext}}-\frac{w_{2}}{2}}-\frac{g}{r_{\mathrm{ext}}-w_{2}-s-\frac{w_{1}}{2}} .
$$

The two additional terms added to the lumped inductance (8) account for the inductive shunt connections between the "signal" and ground. They may be calculated from Rosa's formula for the inductance of rectangular strips [13],

$$
\begin{aligned}
L_{\text {gap } 1 / 2}= & 2\left(w_{1 / 2}+s\right)\left\{\log \left[\frac{2\left(w_{1 / 2}+s\right)}{g+t}\right]+0.5\right. \\
& \left.+0.2235 \frac{g+t}{w_{1 / 2}+s}\right\} .
\end{aligned}
$$

The two additional terms in (7) account for the capacitance between the metal strips at the "gap" or "split" in the SRR. They are approximated by the parallel plate capacitor formula:

$$
C_{\text {gap } 1 / 2}=\varepsilon_{0} \frac{t}{g} w_{1 / 2}
$$

Finally, the resonance frequency of the CSRR is calculated from the standard formula for a resonant LC circuit

$$
\omega_{0}=\frac{1}{\sqrt{\mathrm{LC}}}
$$

From a consideration of Babinet's principle [14], the magnetic polarizability of the SRR [3] and the duality of the SRR and the CSRR [15], we may write the electric polarizability of the CSRR as

$$
\beta_{z z}^{e e}=-\frac{\pi^{2} r_{\mathrm{av}}^{4}}{c^{2} L}\left(\frac{\omega_{0}}{\omega}-1\right)^{-1} .
$$

The subscripts ${ }_{z z}$ mean that the polarizability is due to an excitation pointing in the direction of the normal of the structure, i.e., the $z$ direction (into the page in Fig. 1), and the response acts in the $z$ direction. The superscripts $e e$ mean that both the excitation and the response are electric in character.

The electric dipole moment due to the on-axis $E$ field, $E_{0}$, is

$$
P=\beta_{z z}^{e e} E_{0} .
$$

The electric energy removed from each circular waveguide cell [16], due to each CSRR iris, is given by

$$
\Delta U_{E}=\frac{P}{4}\left(1-e^{-a t} \cos \psi\right) E_{0} .
$$


Here, the term $\alpha t$ represents the attenuation due to propagation through the circular aperture in the CSRR "innerground plane" of thickness $t$ and

$$
\alpha=\sqrt{\left(\frac{2.405}{r_{b p}}\right)^{2}-\left(\frac{\omega}{c}\right)^{2}} \approx \frac{2.405}{r_{b p}} .
$$

$\psi$ is the phase of the particular operating mode and is equal to $k_{z} l$ where $k_{z}$ is the wave number and $l$ is the length of each cell. For a finite number of cells, this quantity takes on discrete values.

We now use Slater's perturbation theorem [17] to relate the operating frequency, $\omega$, to the unperturbed cutoff frequency of the circular waveguide, $\omega_{r}$, via the magnetic energy, $\Delta U_{M}$, and the electric energy, $\Delta U_{E}$, removed from each cell by the two coupling elements. These energies are associated with the aperture magnetic and electric equivalent dipoles:

$$
\frac{\omega-\omega_{r}}{\omega_{r}}=\frac{2\left(\Delta U_{M}-\Delta U_{E}\right)}{U} \quad \text { with } \quad \Delta U_{M}=0 .
$$

The cutoff frequency of the circular waveguide is

$$
\omega_{r}=\frac{2.405 c}{r_{c w}},
$$

and the stored energy associated with the $\mathrm{TM}_{010}$ mode is

$$
U=\frac{\pi r_{c w}{ }^{2} l}{2} \varepsilon_{0} E_{0} 0.5191^{2} .
$$

Combining (13) to (19) we can solve (17) for the resonance frequency. There are three solutions. The one of relevance is given below in (20) through (23).

$$
\begin{gathered}
x_{1}=3 \sqrt{3} \sqrt{-\omega_{0}{ }^{2}\left[4 x_{3}{ }^{3} \omega_{r}+4\left(\omega_{0}{ }^{2}-\omega_{r}{ }^{2}\right)^{2}+x_{3}{ }^{2}\left(\omega_{0}{ }^{2}+12 \omega_{r}{ }^{2}\right)+4 x_{3}\left(5 \omega_{0}{ }^{2} \omega_{r}+3 \omega_{r}{ }^{3}\right)\right]}, \\
x_{2}=2 x_{3}{ }^{3}+9 x_{3} \omega_{0}{ }^{2}+6 x_{3}{ }^{2} \omega_{r}-18 \omega_{0}{ }^{2} \omega_{r}+6 x_{3} \omega_{r}{ }^{2}+2 \omega_{r}{ }^{3} \\
x_{3}=-\frac{23.317 \omega_{r} r_{a v}{ }^{4}\left(1-e^{-\alpha t} \cos \varphi\right)}{r_{c w}{ }^{2} c^{2} l L \varepsilon_{0}} \\
\omega=\Re\left(\frac{x_{3}+\omega_{r}}{3}+\frac{(1-i \sqrt{3})\left[-3 \omega_{0}{ }^{2}-\left(x_{3}+\omega_{r}\right)^{2}\right]}{4.7622\left(x_{1}+x_{2}\right)^{1 / 3}}-\frac{(1+i \sqrt{3})\left(x_{1}+x_{2}\right)^{1 / 3}}{7.56}\right) .
\end{gathered}
$$

Use of (20) through (23) allows one to calculate the dispersion curves for an infinite chain of cells or, for a finite array, the particular mode frequencies. The phase and group velocity can also be obtained by replacing $\psi$ with $k_{z} l$ and, in the case of phase velocity, dividing $\omega$ by $k_{z}$ and, for the group velocity, by differentiating $\omega$ with respect to $k_{z}$. The calculation is straightforward but the formula obtained for the group velocity is extremely long and will not be reproduced here.

We have designed a normal-conducting copper structure with the geometrical parameters given in Table I. The suggested design procedure is as follows. The radius of the circular waveguide, $r_{c w}$, is first selected according to the operational frequency desired. However, the actual operating frequency will vary according to the CSRR parameters and the length of each cell. The parameters associated with the CSRR are chosen such that the resonance frequency is above the cutoff frequency of the circular waveguide as it is found that this condition leads to the largest axial electric field. The value of $w_{2}$ (outer CSRR slot width) can then be increased in order to modify the coupling between cells and further enhance the magnitude of the axial $E$ field without significantly altering the resonance frequency of the CSRRs. The dimensions of the feeding rectangular waveguide are then chosen such that the cutoff frequency of the $\mathrm{TE}_{10}$ mode (controlled by the parameter ' $a$ ') is just below the operational frequency of the structure and the height, $b$, is chosen to be as large as can be comfortably accommodated by the cell length, $l$, as this condition leads to maximum axial electric field.

An eigenmode analysis using CST microwave studio has been performed to study the propagation characteristics of an infinite chain of unit cells defined by the values characterizing the CSRR, feeding waveguide, and circular waveguide given in Table I. Figure 6 shows a plot of the simulated and calculated dispersion characteristics. It can be seen that the dispersion is negative between 0 and $\pi$ degrees phase advance per cell. As previously mentioned, this is in contrast to normal electrically coupled structures in which positive dispersion is obtained. Magnetically coupled structures in which slots are placed in the cell walls towards the outer waveguide walls (where the magnetic fields are appreciable) also display such characteristics. Such structures would have a $\Delta U_{M}$ term in (17). Resonantly coupled structures with appropriate equivalent values of the inductive and capacitive elements can also display negative dispersion. The mode frequencies predicted by the calculations are accurate to about $2 \%$. 
TABLE I. Geometrical parameters for designed structure (see Figs. 1 and 2).

\begin{tabular}{lcc}
\hline \hline Parameter & Value $(\mathrm{mm})$ & Description \\
\hline$a$ & 85 & feeding waveguide width \\
$b$ & 25 & feeding waveguide height \\
$r_{c w}$ & 60 & circular waveguide radius \\
$l$ & 30 & length of each cell \\
$r_{\text {ext }}$ & 12 & external radius of CSRR \\
$w_{2}$ & 5 & outer CSRR slot width \\
$w_{1}$ & 1 & inner CSRR slot width \\
$s$ & 2 & CSRR "signal" width \\
$r_{b p}$ & 3 & hole in CSRR central metal \\
$g$ & 2 & width of CSRR signal/gnd connections \\
$t$ & 1 & thickness of copper \\
\hline \hline
\end{tabular}

Three modes are of particular interest for our present purposes: the $\pi / 3,2 \pi / 3$, and $\pi$ modes as they have phase velocity either close to $c$ or very much below $c$ (see Fig. 7) and can thus be used to accelerate particles.

Figure 7 shows a comparison between calculated and simulated phase velocity. It can be seen that very good agreement is obtained. Note that the phase velocity is very high at low values of phase advance per cell. This means that the 0 mode cannot be used for acceleration purposes as the wave fronts in the accelerating field would quickly overtake an injected particle bunch.

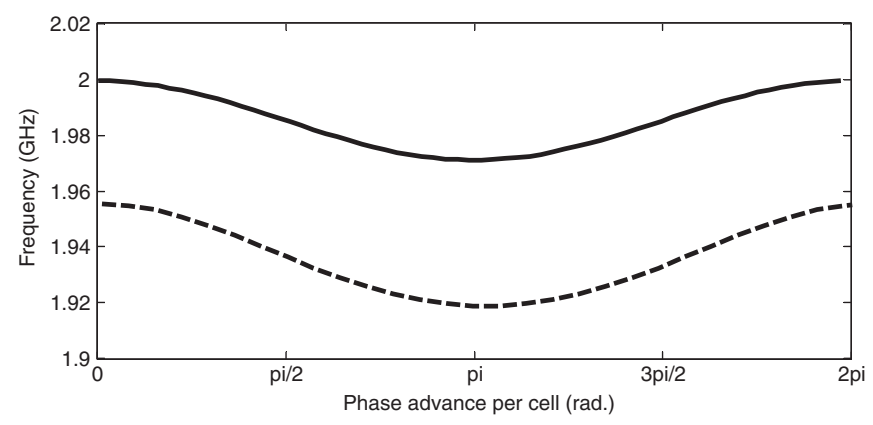

FIG. 6. Dispersion curves for propagating medium consisting of an infinite chain of the unit cells. Calculated: solid line; simulated: square dots.

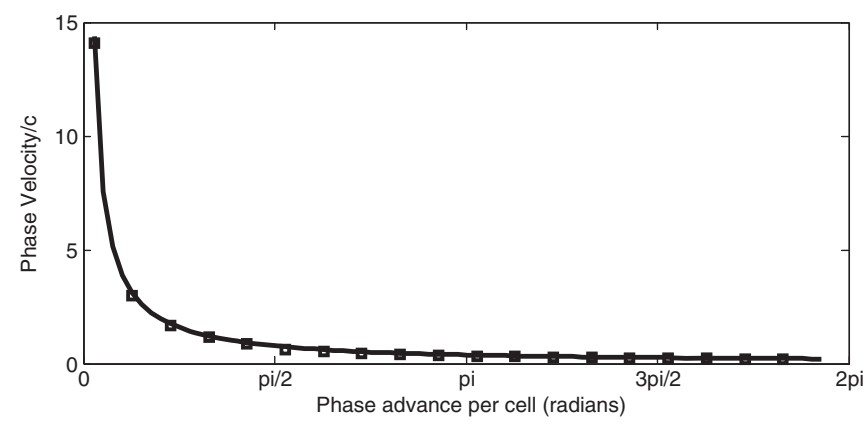

FIG. 7. Calculated and simulated phase velocity for propagating medium consisting of infinite chain of unit cells. Calculated: solid line; simulated: square dots.

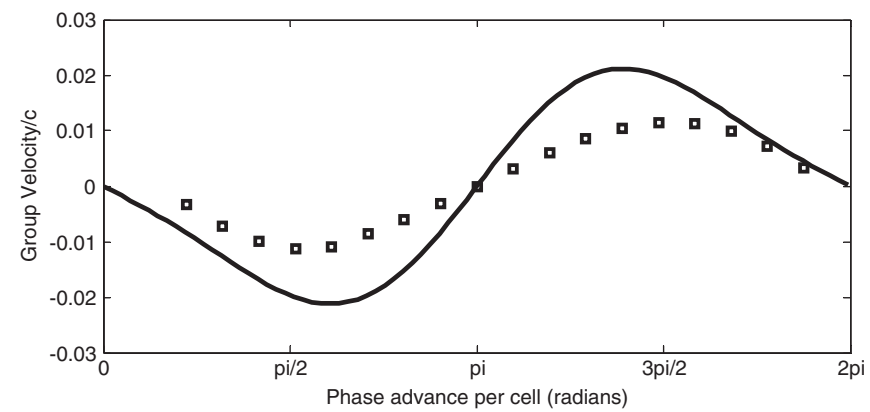

FIG. 8. Calculated and simulated group velocity for propagating medium consisting of an infinite chain of unit cells. Calculated: solid line; simulated: square dots.

Figure 8 shows a comparison between calculated and simulated group velocity. It can be seen that reasonable agreement is obtained. At any one particular value of phase advance per cell, the absolute value of the group velocity is close to the simulated value but the actual shape and slope of the curve is not exact. However, the formulas are good enough to be used as aids in design. The discrepancy could be due to the many simplifications made in the analysis including ignoring the magnetic coupling between cells, the approximate nature of (13) and the fact that the CSRR has been analyzed assuming static current and voltage distributions for the complementary structure-the SRR. The preceding analysis has been checked at various random points in the range $l=12$ to $80 \mathrm{~mm}, r_{\mathrm{ext}}=8$ to $16 \mathrm{~mm}, w_{1} / w_{2}=1$ to $6 \mathrm{~mm}$, and $r_{c w}=40$ to $100 \mathrm{~mm}$, and at all points good agreement for the mode frequencies, phase velocity, and group velocity is obtained. However, it has been noted that when the cutoff frequency of the circular waveguide is below the resonance frequency of the CSRR [as predicted by (12)] the agreement is not good. However, simulations also reveal that maximum accelerating gradient is only achieved when the resonance frequency of the CSRR is some value above the cutoff frequency of the circular waveguide [see (18)].

\section{EM AND PARTICLE SIMULATION RESULTS}

We now turn to EM simulation results for the complete structure (four cells plus end cells and feeding rectangular waveguides-see Fig. 2). All the simulations referred in this section were performed using the frequency domain solver with tetrahedral mesh in CST microwave studio. The structures were first simulated with a frequency guessed to be close to the $\pi / 3$ mode passband. Once the frequency of the $\pi / 3$ mode had been identified, the simulation was rerun with the mesh adaptation set to this frequency. After some trial and error, we settled on a mesh adaptation frequency which, after convergence, yielded a value of $S_{21}$ close to $-4 \mathrm{~dB}$. Figure 9 shows the simulated $S_{21}$ power transmission coefficient. The passbands for the four modes can be clearly distinguished. 


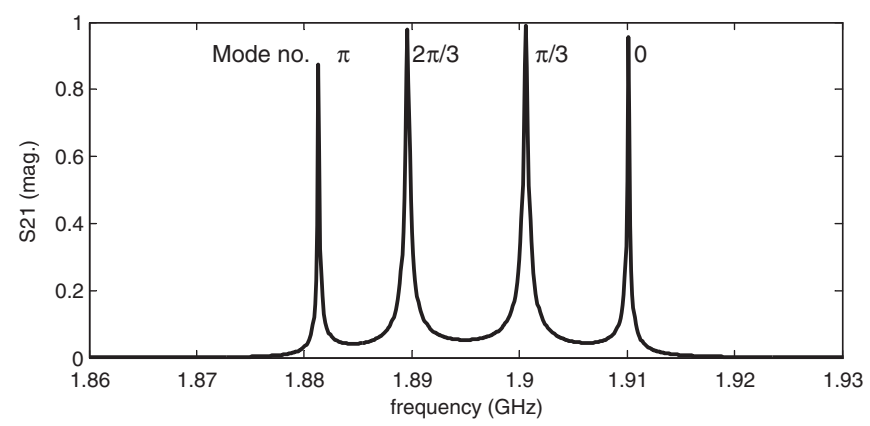

FIG. 9. Plot of the power transmission coefficient between input and output rectangular waveguides.

As indicated earlier, these modes occur in order of decreasing frequency. It can also be seen that the modes are shifted downwards in frequency by about $40 \mathrm{MHz}$ compared with the infinite chain of cells (the difference between calculated values and the $S$-parameter simulation values is around $90 \mathrm{MHz}$ ). This is due to the effect of the end cells and the input and output waveguides.

The fraction of the input power that is lost in the copper walls and irises under steady state conditions may be calculated from the $S$-parameters. Namely,

$$
\text { loss factor }=1-\left|S_{21}\right|^{2}-\left|S_{11}\right|^{2} .
$$

This function is plotted in Fig. 10 with only the three accelerating modes shown.

It can be seen that the power lost is substantial for the $\pi$ mode- $5 \%$ of the applied power is lost in the walls. If $82 \mathrm{~kW}$ were applied, around $4.1 \mathrm{~kW}$ would be dissipated in the structure. Approximately $2 / 3$ of the total power loss is dissipated in the walls of the waveguide and on the "discs" while the remaining $1 / 3$ of the power lost is dissipated in the CSRRs. The CSRRs are not accessible to cooling mechanisms so the structure would have to be operated with an extremely low duty cycle if excessive heating is to be avoided.

Figure 11 shows a plot of the electric field along the axis for the $\pi$ mode. It can be seen that the field is rather nonuniform. It can also be seen that the field is highest in the middle two cells. These features are due to the complex

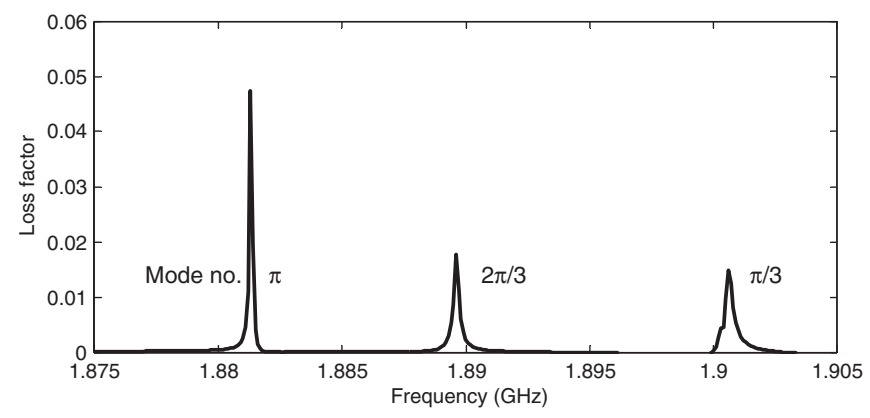

FIG. 10. Plot of the simulated fractional power loss for the complete structure.

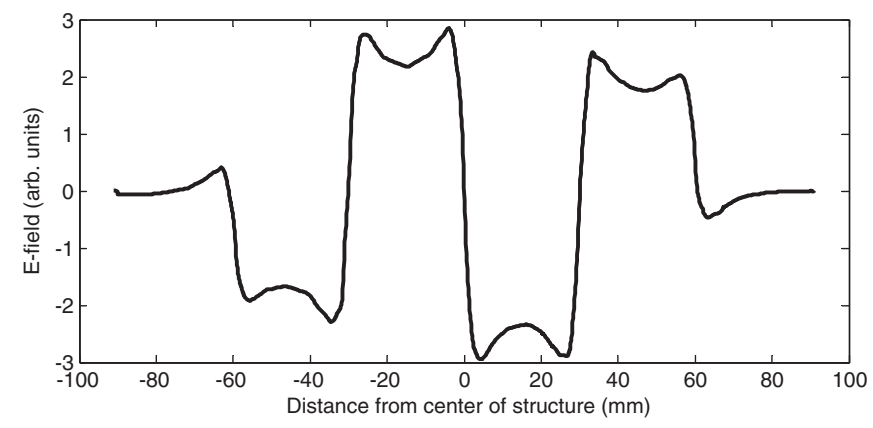

FIG. 11. Plot of the on-axis $E$ field for the $\pi$ mode.

electromagnetic environment caused by the nonsimple geometry of the CSRRs. It is possible that a different type of resonator with a simpler geometry could improve the field "flatness." The main implication of this nonuniformity of field distribution is that the energy spread in the output bunch is increased compared with that of the input bunch. The effect is more pronounced for longer bunches.

At $1 \mathrm{~W}$ input power the peak surface $E$ field for the $\pi$ mode is rather high at $0.13 \mathrm{MV} / \mathrm{m}\left(E_{\text {peak }} / E_{\text {acc }}=11.38\right)$. As might be expected, the peak surface fields occur at the CSRR discontinuities. From the Kilpatrick criterion [18] and Boyd's associated empirical formula for copper surfaces [19], at the operational frequency, the maximum power that may be safely applied to the structure is, approximately, $82 \mathrm{~kW}$. It is common practice to assume that fields twice the value of those predicted by Kilpatrick's criterion can be tolerated since the criterion was formulated before current clean room technology. However, we assume the more conservative values apply as it is possible that our simulations underestimate the peak surface fields-that is, finer and finer meshes applied to the CSRR sections may predict higher fields. Unfortunately, due to constraints on available computing power $(8 \times 64$ bit processors), we were unable to erect arbitrarily fine meshes. The maximum surface electric fields suggested by our simulations for the $2 \pi / 3$ and $\pi / 3$ modes are considerably smaller and the maximum powers that may be safely applied are 0.294 and $0.515 \mathrm{MW}$, respectively. We have conducted particle simulations using these two modes with $2 \mathrm{~mm}$ long, Gaussian-distributed protons bunches with a transverse radius of $2 \mathrm{~mm}$. The results are summarized in Table II. Note that the proton initial energies have been selected to correspond with the phase velocities of the two modes. These particle simulations were used to find the effective accelerating gradient and the corresponding constant, $k$, relating input power to effective accelerating gradient.

Also shown in Table II are three key figures of merit: the cavity $Q$, the shunt impedance per-unit length, and the "filling time," $\tau$. The filling time being of the order of $\mu \mathrm{S}$ means that the structure could be operated in pulsed mode with a consequent increase in the maximum applied rf power and, hence, accelerating gradient [20]. 
TABLE II. Results from particle and EM simulations for the $\pi, 2 \pi / 3$, and $\pi / 3$ modes.

\begin{tabular}{lcccccccc}
\hline \hline $\begin{array}{l}\text { Peak surface field } \\
(\mathrm{MV} / \mathrm{m} \text { @ 1 W }\end{array}$ & $\begin{array}{c}\text { Applied } \\
\text { power (MW) }\end{array}$ & $\begin{array}{c}\text { Proton initial } \\
\text { energy }(\mathrm{MeV})\end{array}$ & $\begin{array}{c}\text { Operating } \\
\text { mode }\end{array}$ & $\begin{array}{c}\text { Effective } \\
\text { accelerating } \\
\text { gradient }(\mathrm{MV} / \mathrm{m})\end{array}$ & $\begin{array}{c}k,(\text { effective accelerating } \\
\text { gradient }(\mathrm{MV} / \mathrm{m}))=k \\
\sqrt{ } \text { AppliedPower(MW) }\end{array}$ & $\begin{array}{c}R_{\text {shunt }} \\
(\mathrm{M} \Omega / \mathrm{m})\end{array}$ & $\tau_{f}(\mu \mathrm{s})$ \\
\hline 0.13 & 0.082 & 80 & $\pi$ & 3.27 & 11.4 & 9213 & 40.8 & $\ldots$ \\
0.09 & 0.1785 & 201 & $2 \pi / 3$ & 3.37 & 8 & 9949 & 21.2 & 1.67 \\
0.07 & 0.297 & 2000 & $\pi / 3$ & 3.06 & 5.62 & 11025 & 38.16 & 1.83 \\
\hline \hline
\end{tabular}

By way of comparison with conventional structures, a standard disc-loaded waveguide was simulated whose geometry was identical to that in Table I except that the CSRR was removed-that is, a structure that is a discloaded waveguide with an iris radius of $12 \mathrm{~mm}$. While it is true that the shunt impedance decreases as the iris radius is increased and that it might therefore be argued a valid comparison cannot be made between a CSRR-based structure with an iris of $3 \mathrm{~mm}$ and a disc-loaded structure with iris diameter of $12 \mathrm{~mm}$, it was found that structures with iris radii of less than $12 \mathrm{~mm}$ (especially $3 \mathrm{~mm}$ ) did not allow successful wave propagation from the input to output port. Therefore, our CSRR-based structure can only be compared with a structure whose iris radius is $12 \mathrm{~mm}$ or larger, when all other geometrical parameters remain the same. It was found that the accelerating gradients for the $\pi, 2 \pi / 3$, and $\pi / 3$ modes in the CSRR structure were $3.3,1.27$, and 1.6 times those of the corresponding modes in the conventional structure. The shunt impedances of the CSRR structure were found to be $2.8,1.1$, and 2.2 times those in the conventional structure. Thus, for a fixed (low) input power, the CSRR structure produces a larger accelerating gradient than does the conventional disc-loaded structure.

Such accelerating gradients do not, however, compare well with the accelerating gradients of around $31.5 \mathrm{MV} / \mathrm{m}$ that are routinely achieved by superconducting cavities operating at $1.3 \mathrm{GHz}$ [21]. However, copper cavities do not require such elaborate cooling systems as superconducting cavities and are generally easier and cheaper to fabricate.

One of the main disadvantages of our structure compared with the conventional one is that the aperture for the

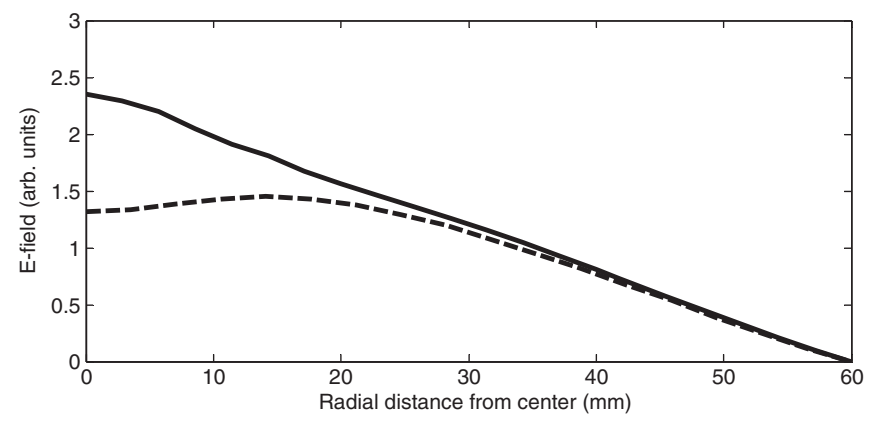

FIG. 12. Plot of the axial $E$ field in the middle of one of the cells along a radial line for the conventional circular aperture structure (broken line) of iris diameter $12 \mathrm{~mm}$ and the CSRR-loaded structure (continuous line). particle bunch is considerably smaller. This is a disadvantage that becomes more pronounced as the operational frequency is made higher and the corresponding CSRR dimensions shrink. This may have implications for the wakefield effect and bunch instability. Another disadvantage is that the CSRR-loaded structures tend to have much higher peak surface electric fields. The consequence of this is that lower excitation powers can cause $\mathrm{rf}$ breakdown. However, as we have seen, lower powers are sufficient for the CSRR structure to achieve the same accelerating gradients exhibited by conventional ones. Thus the CSRRloaded structure is more efficient at translating input $\mathrm{rf}$ power into accelerating gradients.

A physical explanation for the increased accelerating gradient exhibited by the CSRR-loaded structure can be given in terms of the distribution of the accelerating field along a radial line from the center of the structure. Figure 12 shows a plot of the $E$ fields in both the conventional and CSRR-loaded structure in the middle of one of the cells. The $x$ axis in this plot represents the distance along a radial line from the center of the structure, i.e., from one side of the metal wall in the circular waveguide to the opposite point in the metal wall along a line that passes through the center of the structure.

It can be seen that the $E$ field of the conventional design levels off some distance from the center of the structure and experiences a dip at the actual midpoint. In contrast, the field in the CSR- loaded structure continues to increase and reaches a peak in the very center of the structure.

\section{CONCLUSIONS}

We have described a novel traveling wave structure in which CSRRs are used to couple pillbox cavities. It has been shown, theoretically, that the ordering of the 0 through $\pi$ modes decreases in frequency. Radio-frequency breakdown is a potential problem but input powers of around $0.082 \mathrm{MW}$ ( $\pi$ mode), $0.1785 \mathrm{MW}(2 \pi / 3$ mode), and $0.297 \mathrm{MW}(\pi / 3$ mode) should be possible. With such powers, our simulations suggest that an accelerating gradient as high as $3.37 \mathrm{MV} / \mathrm{m}$ and a cavity voltage of around $0.6 \mathrm{MV}$ is possible at $1.9 \mathrm{GHz}$. The basic concepts proposed in this paper may be adapted to more advanced technologies, including the use of nose cones, elliptical cross sections for the unit cells, and standing wave modes. It may also be possible to use coupling elements with a simpler geometry or rounded off discontinuities that 
produce lower peak surface fields than the complicated CSRR geometries and, hence, increase the maximum safe applied power.

\section{ACKNOWLEDGMENTS}

We thank the Science and Technology Facilities Council, United Kingdom, for financial support.

[1] E.J.N. Wilson, Introduction to Particle Accelerators (Oxford University Press, New York, 2001), Chap. 10.

[2] J. B. Pendry, A. J. Holden, D. J. Robbins, and W. J. Stewart, IEEE Trans. Microwave Theory Tech. 47, 2075 (1999).

[3] R. Marques, F. Martin, and M. Sorolla, Metamaterials with Negative Parameters-Theory, Design and Microwave Applications (John Wiley and Sons, New York, 2008), Chap. 1.

[4] D. Schurig, J. J. Mock, B. J. Justice, S. A. Cummer, J. B. Pendry, A. F. Starr, and D. R. Smith, Science 314, 977 (2006).

[5] M. A. Shapiro, S. Trendafilov, and Y. Urzhumov, Phys. Rev. B 86, 085132 (2012).

[6] Y. S. Tan and R. Seviour, Europhys. Lett. 87, 34005 (2009).

[7] S. Antipov, L. Spentzouris, W. Gai, W. Liu, and J. G. Power, Nucl. Instrum. Methods Phys. Res., Sect. A 579, 915 (2007).

[8] F. Falcone, T. Lopetegi, J. D. Baena, R. Marques, F. Martin, and M. Sorolla, IEEE Microw. Wirel. Compon. Lett. 14, 280 (2004).
[9] S. Antipov, L. Spentzouris, W. Gai, M. Conde, and F. Franchini, J. Appl. Phys. 104, 014901 (2008).

[10] M. A. Shapiro, J.R. Sirigiri, and R. J. Temkin, in Proceedings of the 23rd Particle Accelerator Conference, Vancouver, Canada, 2009 (IEEE, Piscataway, NJ, 2009).

[11] Y. Shin, 36th International Conference on Infrared, Millimeter and Terahertz. Waves (IRMMW-THz), 2011 (IEEE, 2011), http://ieeexplore.ieee.org/xpls/abs_all.jsp? arnumber $=6105234 \& \operatorname{tag}=1$.

[12] S. S. Gevorgian and I. G. Mironenko, Electron. Lett. 26, 1916 (1990) [http://ieeexplore.ieee.org/application/ enterprise/entconfirmation.jsp?arnumber $=59503$ \&icp=false].

[13] E. B. Rosa, Bulletin of the Bureau of Standards 4, 301 (1908), Scientific Paper 80 (S80).

[14] R. F. Harrington, Time-Harmonic Electromagnetic Fields (McGraw-Hill, New York, 1961).

[15] J.D. Jackson, Classical Electrodynamics (Wiley, New York, 1999) 3rd. ed.

[16] T. Wangler, RF Linear Accelerators (Wiley and Sons, New York, 1998).

[17] J.C. Slater, Microwave Electronics (D. Van Nostrand Company, New York, 1950), pp. 80-81.

[18] W. D. Kilpatrick, Rev. Sci. Instrum. 28, 824 (1957).

[19] T. J. Boyd, Jr., Los Alamos Group AT-1 Report No. AT-1:82-28, 1982.

[20] G. A. Leow and J.W. Wang, Report No. SLAC-PUB5059, 1989.

[21] H. Hitomi, F. Inoue, H. Hara, K. Sennyu, K. Kanaoka, and T. Yanagisawa, Proceedings of the 2nd International Particle Accelerator Conference, San Sebastián, Spain (EPS-AG, Spain, 2011). 